\title{
MENTE X CORAÇÃO: AS DOENÇAS PSIQUICAS E AS CONSENQUÊNCIAS CARDIOVASCULARES: UMA REVISÃO DA LITERATURA
}

\author{
MIND X HEART: PSYCHIC DISEASES AND CARDIOVASCULAR \\ CONSENQUENCIES: ALITERATURE REVIEW
}

Beatriz da Silva Araújo ${ }^{1}$

Morgana Gonçalves da Silva ${ }^{2}$

\begin{abstract}
RESUMO: OBJETIVO: O presente estudo objetiva conhecer as principais doenças psíquicas que afetam as funções cardiovasculares e as consequências para o coração, visto que, para a Organização Mundial da Saúde, problemas cardiovasculares são considerados um dos contribuidores para pessoas que sofrem de doenças mentais potencialmente severas. METODOLOGIA: Trata-se de uma revisão integrativa de literatura, com abordagem qualitativa. Realizada por busca eletrônica nas seguintes bases de dados: PUBMED, MEDLINE e SCIELO. Foram utilizados os seguintes descritores: Transtornos de Ansiedade; Doenças Cardiovasculares; Depressão; Saúde Mental. Foram selecionados artigos publicados no período de 2015 a 2019, disponíveis na integra e em língua portuguesa e inglesa, que, ao final da pesquisa, totalizaram 13 artigos. RESULTADOS: A Ansiedade está associada a alguns comportamentos maléficos à saúde, como o tabagismo, álcool e diminuição de atividade física, o que potencializa o risco de o sujeito apresentar uma DCV. Além disso, evidência existente sugere que a relação entre saúde mental e risco de DCV é mais forte entre os indivíduos que atendem aos critérios de diagnóstico para um Transtorno Depressivo Maior e Transtorno Bipolar. CONCLUSÃO: Os indivíduos que sofrem com doenças psíquicas, devem ter um atendimento mútuo, entre psicólogos/psiquiatras e cardiologistas, pois como mostram os estudos analisados, a partir de fatores predisponentes, as doenças psíquicas podem gerar riscos cardiológicos, tendo grande potencial de afetar, inclusive, o decaimento da condição mental do indivíduo.
\end{abstract}

Palavras chave: Doenças Cardiovasculares. Depressão. Saúde Mental. Transtornos de Ansiedade

\footnotetext{
${ }^{1}$ Graduanda do Curso de Enfermagem da Unifacisa.

${ }^{2}$ Graduanda do Curso de Enfermagem da Unifacisa.
} 
ABSTRACT: OBJECTIVE: The present study aims to know the main psychic diseases that affect cardiovascular functions and the consequences for the heart, since, for the World Health Organization, cardiovascular problems are considered one of the contributors for people suffering from potentially mental illnesses severe. METHODOLOGY: This is an integrative literature review, with a qualitative approach. Performed by electronic search in the following databases: PUBMED, MEDLINE and SCIELO. The following descriptors were used: Anxiety Disorders; Cardiovascular diseases; Depression; Mental health. Articles published in the period from 2015 to 2019 were selected, available in full and in Portuguese and English, which, at the end of the research, totaled 13 articles. RESULTS: Anxiety is associated with some harmful behaviors to health, such as smoking, alcohol and decreased physical activity, which increases the risk of the subject having CVD. In addition, existing evidence suggests that the relationship between mental health and CVD risk is strongest among individuals who meet the diagnostic criteria for Major Depressive Disorder and Bipolar Disorder. CONCLUSION: Individuals who suffer from psychic illnesses should have mutual assistance, between psychologists / psychiatrists and cardiologists, as shown by the analyzed studies, based on predisposing factors, psychic illnesses can generate cardiological risks, with great potential to affect, including, the decay of the individual's mental condition.

Keywords: Cardiovascular diseases. Depression. Mental health. Anxiety Disorders. 


\section{INTRODUÇÃO}

No Brasil, as doenças cardiovasculares representam as principais causas de mortes. De acordo com o Ministério da Saúde, cerca de 300 mil indivíduos por ano sofrem Infarto Agudo do Miocárdio (IAM), ocorrendo óbito em $30 \%$ desses casos. Estima-se que até 2040 haverá aumento de até $250 \%$ desses eventos no país. (BRASIL, 2020).

O Brasil é um país de dimensões continentais, com uma das maiores desigualdades socioeconômicas, uma situação que inevitavelmente está relacionada com maior mortalidade por doenças não transmissíveis, especialmente doença cardiovascular (DCV)(POLANCZYK, 2020).

De acordo com Almeida et al., a doença mental surge como uma barreira que dificulta o contato do indivíduo com o ambiente em que está inserido, tornando-o alienado e na maioria das vezes privando-o de sua liberdade e da possibilidade do convívio com as pessoas. Para Espinosa (2008), o transtorno mental pode ser entendido como uma alteração fisiológica ou orgânica e psicológica, ou ainda um desequilíbrio emocional causado por fatores externos ou internos.

Almeida et al., compreende que a doença mental significa modificar e desconstruir ideologias, crenças e valores em relação a patologias mentais, utilizando princípios norteadores do processo de transformação institucional através da Reforma Psiquiátrica, Lei n.o 10.216 instituída em 06 de abril de 2001, que tem como proposta transformar o modelo assistencial de Saúde Mental através da construção de um novo estatuto social para pessoas portadoras de transtornos mentais respeitando os princípios fundamentais de cidadania.

De acordo com Correia e Sougley (2017), a depressão é uma doença de expressão clínicacomplexa que altera particularmente o humor. Acomete o indivíduo em sua totalidade, provocando grande sensação de desconforto e sofrimento. A depressão também produz vários sintomas físicos (vividos corporalmente), como a sensação de cansaço/fadiga, lentificação geral, perturbação do sono, perda de peso 
e diminuição das apetências. Compõe-se assim um intricado conjunto sindrômico que atinge o indivíduo como um todo, provocando grande sensação de mal-estar.

A depressão é altamente prevalente em pacientes com doença cardiovascular (DCV) e prenuncia resultados cardiovasculares adversos e aumento dos custos de saúde. Um em cada 5 pacientes com doença arterial coronariana ou insuficiência cardíaca está deprimido, uma prevalência que é pelo menos 3 vezes maior do que na população em geral (COHEN, 2015).

Na visão de Pretribu e Melo (2017), a ansiedade pode ser definida como uma sensaçãoinquietante vaga e difusa, desagradável, de apreensão expectante negativa em relação ao futuro, que se acompanhada de diversas manifestações físicas (dispneia, taquicardia, tensão muscular, tremores, sudorese, tontura etc.) e, até certo ponto, é um estado afetivo normal e útil. Os transtornos de ansiedade (TA) surgem quando a ansiedade excede o limite da normalidade, de modo que tal sensação se torna tão intensa e desagradável que impede o funcionamento adequado do indivíduo.

Segundo a Organização Mundial da Saúde (2018), problemas cardiovasculares são considerados um dos contribuidores para pessoas que sofrem de doenças mentais potencialmente severas. O risco de uma pessoa com doença mental ter problemas cardiovasculares está aproximadamente entre um a três vezes maior, se equiparado a pessoas sem desordens mentais, isso contribui para o aumento do índice de morbidade e mortalidade, comparada a população geral.

A Organização Mundial da Saúde afirma que o uso de medicamentos antipsicóticos foi associado a obesidade, resistência à insulina, diabetes, infecções miocárdicas, fibrilação atrial e morte, tornando-se clara a premissa que desordens mentais, podem afetar diretamente em problemas cardiovasculares futuros do sujeito. 


\section{METODOLOGIA}

Esse estudo trata-se de uma revisão integrativa da literatura sobre as doenças psíquicas (transtornos de ansiedade, depressão, transtorno de pânico etc.) e sua relação com doenças cardiovasculares. Foram utilizados os descritores em português encontrados no DeCS (Descritores em Ciência e Saúde) "Transtornos de Ansiedade"; "Doenças Cardiovasculares"; "Depressão"; Transtorno de Pânico" e "Saúde Mental|".

O critério de exclusão foi adotado para artigos no qual não condiziam com o objetivo proposto, aqueles que não abordavam as doenças mentais e a relação com a cardiologia, além de exclusão de trabalhos que tinham data de publicação inferior ao ano de 2015 e os que abordavam as doenças psíquicas como causa secundaria a um problemas cardiovascular.

$\mathrm{Na}$ base de dados MEDLINE foi utilizada as palavras chave: Doenças Cardiovasculares, Transtorno de Ansiedade e Depressão, foram encontrados 160 artigos, no qual desses foram selecionados 3 de acordo com os critérios de exclusão citados.

$\mathrm{Na}$ base de dados SCIELO foram encontrados 17 artigos, seguindo os critérios de exclusão, selecionou-se ao final 2 artigos.

$\mathrm{Na}$ base de dados PubMed, utilizando as chaves Cardiovascular Disease AND Anxiety Disorders AND Depression, foram encontrados 811 artigos de língua portuguesa e inglesa. Após a aplicação de filtros, a parti do ano de 2015 e full text ficaram 438. Após análise dos artigos encontrados foram excluídos aqueles que não abordavam o assunto de interesse para a revisão, finalizando 8 artigos lidos na integra. 


\section{RESULTADOS E DISCUSSÃO}

Os 13 artigos utilizados para esse estudo evidenciam que as pessoas no qual apresentam tais doenças psíquicas como, transtornos de ansiedade, depressão, influencia para desencadearproblemas cardiovasculares futuros. Dessa forma, serão apresentados os desfechos dos estudos de modo que, será dividido de acordo com as pesquisas realizadas, mostrando os fragmentos relevantes, contribuindo pra a compreensão desse artigo de revisão.

No estudo realizado por Cohen e colaboradores (2015) é abordado alguns mecanismos no qual pode influenciar o início ou progressão de DCV. Nesse, os autores afirmam que a ansiedade está associada a alguns comportamentos maléficos à saúde, como o tabagismo, álcoole diminuição de atividade física, o que potencializa o risco de o sujeito apresentar uma DCV. Cohen exprimi um modelo biológico, onde explica a fisiopatologia da ansiedade, onde a mesma aumenta a liberação de catecolaminas pelo sistema nervoso, dano endotelial, consequentemente levando à arteriosclerose. Além disso, o estudo mostra que o estresse é outro fator de risco, por aumento da frequência cardíaca em repouso, maior variabilidade de repolarização ventricular, sendo assim, esses aumentam o risco de o indivíduo apresentar arritmias cardíacas, infarto agudo do miocárdio e morte cardíaca súbita.

Embora os transtornos de ansiedade possam contribuir para o risco cardiovascular e possam definir o terreno para eventos agudos desencadeados emocionalmente em particular, atualmente nenhum estudo demonstrou se o tratamento de transtornos de ansiedade compensa o risco cardiovascular (COHEN, 2015).

Um estudo transversal com participantes entre idade de 8 a 18 anos avaliou a saúde cardiovascular em relação ao percentual de gordura corporal relacionando a doenças mentais, Gross e contribuintes (2017), relatam que o estresse é aumentado em pessoas com transtornos mentais, pois os mesmos podem ter piora da função autonômica, portanto conforme aumenta adepressão essa função fica vulnerável. 0 
efeito cumulativo dessas alterações fisiopatológicas associadas a problemas de saúde mental pode acelerar a progressão da DCV (KAIZER, 2017).

A evidência existente sugere que a relação entre saúde mental e risco de DCV é mais forte entre os indivíduos que atendem aos critérios de diagnóstico para um Transtorno Depressivo Maior e Transtorno Bipolar (KAIZER, 2017).

Contudo, a ansiedade não foi significativamente associada a fatores de risco de DCV ou saúde vascular em nenhum dos modelos. A depressão foi associada ao colesterol HDL, triglicerídeos e pontuação do grupo da síndrome metabólica, essas relações foram atenuadas aocontabilizar o percentual de gordura corporal (GROSS et al., 2017).

Outro estudo de coorte transversal, objetivou a analisar e estimar a prevalência de transtornos de ansiedade e depressão em pacientes de atenção primária e associar com fatores cardiovasculares. Houve associação significativa entre transtornos ansiosos e depressivos e hipertensão, obesidade e hiperlipidemia, mesmo quando ajustados para idade, sexo e variáveis socioeconômicas, além disso os indivíduos do grupo depressão e ansiedade apresentaram risco adicional de hipertensão arterial (VELOSO et al., 2019).

Perez e colaboradores (2020), em seu estudo de coorte mostram evidências do risco cardiovascular em pacientes com transtornos psiquiátricos. Os antidepressivos foram associados a maior risco de diabetes, hipertensão e hiperlipidemia. Os antipsicóticos foram associados a um maior risco de diabetes. Antidepressivos e antipsicóticos foram associados a menor risco de outros fatores de risco. Pacientes com doenças psiquiátricas têm detecção posterior de fatores de risco cardiovascular (PEREZ et al., 2020).

Meng e contribuintes (2020), realizara um estudo de coorte afim de investigar a depressão como um fator de risco para causas de mortalidade cardiovascular em adultos chineses. A taxa de incidência de mortalidade cardiovascular e por todas as causas entre os participantes com depressão foi significativamente maior do que entre aqueles sem depressão em ambas as coorte. Com isso, a depressão foi um fator de risco independente de mortalidade por todas as causas e mortalidade cardiovascular em adultos chineses, especialmente em homens. Mais estudos com depressão clinicamente diagnóstica e medidas repetidas de depressão ainda são 
necessários para confirmar achados em populações chinesas e esclarecer os potenciais mecanismos subjacentes (MENG et al., 2020).

Marc, Johan e Davy em seus estudos sobre a relação entre doença coronariana e transtornos mentias, afirma que essas estão entre as principais causas de morbidade e mortalidade em todo o mundo. Embora os resultados de revisões e metanálises mais antigas, pesquisadas e analisadas por os autores, tenham sido inconsistentes, as posteriores, incluindo dados mais rigorosos e prospectivos, mostraram que a depressão constitui um fator de risco. Deacordo com uma metanálise de 30 estudos de coorte prospectivos, indivíduos com depressão, em comparação com pessoas não deprimidas, experimentam um risco aumentado significativo de 30\% para Doença Isquêmica do Coração (DIC). Outra metanálise de estudos de coorte prospectivos, analisado pelo os autores descobriu que a depressão estava associada a um aumento de $31 \%$ no risco de infarto do miocárdio e um aumento de $36 \%$ no risco de morte coronária, em comparação com pessoas não deprimidas (DE HERT, 2018).

Marc e colaboradores ainda relatam que os sintomas ou transtornos de ansiedade podemestar associados a um risco aumentado de leve a moderado de desenvolver Doenças Coronarianas (DCC). Três metanálises, reforçaram os achados de estudos anteriores de que a ansiedade (transtornos) pode estar associada a DCC incidente. De acordo com o mais recente, a ansiedade estaria associada a um risco $41 \%$ maior de desenvolver DIC. A ansiedade fóbica foi mais fortemente associada à DCC incidente do que a ansiedade geral / não especificada. Outra metanálise de 20 estudos, avaliando a associação de ansiedade, ou seja, pânico, fobia e preocupação, com a DAC incidente, descobriram que indivíduos inicialmente saudáveis com alta ansiedade tinham um risco $26 \%$ maior de DIC (DE HERT, 2018).

Hert ainda afirma em seu estudo que o diferencial de mortalidade em pessoas com transtornos mentais não decorre apenas de uma série de fatores comportamentais e de estilo devida, mas também de fatores biológicos que parecem ser comuns tanto a fatores mentais quantodoenças cardio-metabólicas. 


\section{CONCLUSÃO}

As doenças psíquicas em conjunto com as comorbidades cardiovasculares estão entre as maiores causas de morbidade e mortalidade no Brasil. A partir dessa revisão de literatura e análise dos artigos estudados, a ansiedade, depressão e estresse, mostram-se como os mais prevalentes predispostos para o indivíduo desenvolver-se futuramente doenças cardiovasculares, tendo a depressão como o transtorno mais citado entre os artigos selecionados.

Além disso, os principais motivos para as doenças mentais estarem relacionadas com problemas cardiovasculares está no fato de que as mesmas geram no sujeito uma mudança no seu quadro fisiológico, fazendo com que o sistema cardiovascular fique vulnerável. Assim como afirma Cohen (2017) em seu estudo, as catecolaminas liberadas na durante uma crise de ansiedade causam danos no endotélio, que consequentemente poderá gerar no sujeito uma arterosclerose e posteriormente evoluir com infarto do miocárdio.

Nesse contexto, é necessário o acompanhamento profissional mútuo, ou seja, psicólogo/psiquiatra e cardiologista, entre indivíduos que apresentam doenças psíquicas, pois as mesmas, a partir de fatores predisponentes, podem gerar riscos cardiológicos, podendo afetarinclusive o decaimento de sua condição mental.

\section{REFERÊNCIAS BIBLIOGRÁFICAS}

COHEN, B.; EDMONDSON, D.; KRONISH, I. Revisão do estado da arte: depressão, estresse, ansiedade e doença cardiovascular. American Journal of Hypertension. Vol. 28. 2015.

GROSS, A. et al. "Relações de ansiedade e depressão com saúde cardiovascular em jovens com peso normal para obesidade severa". The jornal of pediatrics. vol. 199, p. 85-91. 2018. Disponível em: https://www.ncbi.nlm.nih.gov/pmc/articles/PMC6063783/\#R2. Acesso em: 03de fevereiro de 2021.

KARATAS, M.B et al. Ansiedade, Depressão e Distúrbios Psicológicos Gerais em Pacientes com Fluxo Coronariano Lento. Arq. Bras. Cardiol., São Paulo, v. 105, n. 4, p. 362-370, out. 2015.

http://www.scielo.br/scielo.php?script=sci_arttext\&pid=S0066782X2015002300362\&lng=pt \&nrm=iso. Acesso em: 03 de fevereiro de 2021. 
LEMOS, M.A; AGUDELO, D.M. Considerações sobre a depressão como fator de risco nos pacientes cardiovasculares. CES Psicol. Medellín, v. 8, n. 1, pág. 192-206, junho de 2015. Disponível

em: http://www.scielo.org.co/scielo.php?script=sci_arttext\&pid=S201130802015000100013\&lng=en \&nrm=iso acesso em 03 de fevereiro de 2021.

MENG, R. et al. Associação entre depressão e mortalidade cardiovascular e por todas as causas em adultos chineses. Jama network open. vol. 3, p.2, 2020.

PÉREZ, M. et al. Fatores de risco cardiovascular entre pacientes com esquizofrenia, transtornos bipolares, depressivos, de ansiedade e de personalidade. European Psychiatry, vol.35, p.815,2016 .

POLANCZYK, C.A. Epidemiologia das Doenças Cardiovasculares no Brasil: A verdade escondida nos números. ABC Cardiol. 2020. Disponível em: http://abccardiol.org/wpcontent/plugins/xml-to-html/include/lens/index.php?xml=0066-782X

abc115020161.xml\&lang=pt-br\#info. Acesso em 03 de fevereiro de 2021.

VELOSO, M.A.O et al. Prevalência de depressão e ansiedade e sua associação com fatores de risco cardiovascular em pacientes da atenção primária do Nordeste do Brasil. Rev. Assoc. Med. Bras., São Paulo, v. 65, n. 6, pág. 801-809, junho de 2019. Disponível em: http://www.scielo.br/scielo.php?script=sci_arttext\&pid=S010442302019000600801\&lng=en \&nrm=iso. Acesso em 03 de fevereiro de 2021.

MONTEIRO, A. Psiquiatria clínica. MedBook Editora, Rio de Janeiro, 2017. Disponível em: https://integrada.minhabiblioteca.com.br/\#/books/9786557830031/. Acesso em: 04 de fevereiro de 2021.

PARK, K.S et al. Associations of depression and anxiety with cardiovascular risk among people living with HIVIAIDS in South Korea. Epidemiol Health. 2020.

MALTA, D.C et al. Mortalidade por doenças não transmissíveis no Brasil, de 1990 a 2015, segundo estimativas do estudo Global Burden of Disease. São Paulo Med. J., São Paulo, v. 135, n. 3, pág. 213-221, junho de 2017. Disponível em: http://www.scielo.br/scielo.php?script=sci_arttext\&pid=S151631802017000300213\&lng=en \&nrm=iso. Acesso em 04 de fevereiro de 2021.

CELANO, C.M et al. Anxiety Disorders and Cardiovascular Disease. Curr Psychiatry Rep. 2016. Disponível em: https://pubmed.ncbi.nlm.nih.gov/33445826/. Acesso em: 03 de fevereirode 2021. 